DETC2007-35832

\title{
TOWARDS AN INTERACTIVE ASSESSMENT FRAMEWORK FOR ENGINEERING DESIGN LEARNING
}

\author{
Carolyn P. Rosé \\ Carnegie Mellon University \\ Language Technologies Institute and \\ Human-Computer Interaction Institute \\ Pittsburgh, Pennsylvania 15213 \\ cprose@cs.cmu.edu \\ Jaime Arguello \\ Carnegie Mellon University \\ Language Technologies Institute \\ Pittsburgh, Pennsylvania 15213 \\ jarguell@andrew.cmu.edu \\ Asim Smailagic \\ Carnegie Mellon University \\ Dept. of Civil \& Environmental \\ Engineering and Institute for Complex \\ Engineered Systems \\ Pittsburgh, Pennsylvania 15213 \\ asim@cs.cmu.edu
}

\author{
Gahgene Gweon \\ Carnegie Mellon University \\ Human-Computer Interaction Institute \\ Pittsburgh, Pennsylvania 15213 \\ ggweon@cs.cmu.edu
}

\author{
Susan Finger \\ Carnegie Mellon University \\ Dept. of Civil \& Environmental Engineering \\ and Institute for Complex Engineered \\ Systems \\ Pittsburgh, Pennsylvania 15213 \\ sfinger@ri.cmu.edu \\ Daniel P. Siewiorek \\ Carnegie Mellon University \\ Electrical and Computer Engineering and \\ Human-Computer Interaction Institute \\ Pittsburgh, Pennsylvania 15213 \\ dps@cs.cmu.edu
}

\begin{abstract}
In this paper we explore the use of text processing technology for on-line assessment in an engineering design project class. We present results from a 5-week classroom study in a capstone engineering design course in which we explore the potential benefits of such technology for student learning in this context. Furthermore, we present results from ongoing work assessing student productivity based on features extracted from their conversational behavior in the course discussion board. While we found that typical shallow productivity measures such as number of posts, length of posts, or number of files committed have no correlation with an instructor assigned grade, we can achieve a substantial improvement using simple linguistic patterns extracted from on-line conversational behavior.
\end{abstract}

Keywords: design education, design teams, design communication

\section{INTRODUCTION}

Project-based learning, especially in courses where students work in groups on real world problems for industry sponsors, is commonly believed by educators and administrators alike to have great value for engineering students (Dutson et al. 1997; Adams 2001). These courses are often situated in engineering curricula as capstone design courses that offer students the opportunity to integrate and apply the knowledge they have acquired in more theoretical courses. Furthermore, these project courses are highly valued by students in engineering departments who seek authentic experiences in their field of choice.

Multi-disciplinary design project classes present challenges both for supporting and for assessing learning because the learning is self-directed and knowledge is acquired as needed throughout the design process. Thus, not all of the students are learning the same thing at the same time. 
Furthermore, the bulk of student learning takes place without the instructor present. While this provides students with opportunities to develop skills related to the ABET goal of lifelong learning, it can have several negative consequences. One is that students often flounder out of view of the instructor and may not have adequate support from their team mates. Students may not know how to begin to construct their own knowledge or they may go off on a technical tangent based on incorrect initial assumptions. Instructors are often unaware when intervention would be beneficial to an individual or to a team. Another difficulty when learning is self-directed is that each student learns a different set of knowledge, so assessing learning is difficult Because the instructor does not direct the learning, the instructor often has difficulty assessing what students have learned - both in terms of technical skills and professional skills.

As instructors of design courses, our recurring frustration has been the observation that rather than focus on learning as much as possible from their project, students often focus on the result, which often leads them in directions that are not conducive to their learning. Faculty who are teaching design classes have the insights necessary to help students learn from the experiences they are having, but do not know when the students need guidance. The over-arching goal of our research has been to work towards shifting the focus in design courses from producing a product to supporting learning. The research contribution of this paper is a description of our work in progress towards developing an infrastructure to obtain an evidence base for reforming engineering design learning in project courses.

In this paper, we present the methodological framework for our research. We then describe the engineering design course that has been the testbed for our work. Next we describe a qualitative analysis of data collected in this course, which offers supporting evidence of our claim that students in design courses tend to focus on performance rather than learning. We describe an experimental study that offers some evidence that, if instructors were able to offer in-process, targeted instruction to their students, it would have a lasting effect. We conclude with results from work in progress on an on-line assessment infrastructure designed to offer instructors insight into team processes that are an integral part of design courses.

\section{RESEARCH AGENDA: STUDYING AND SUPPORTING ENGINEERING DESIGN LEARNING}

Obtaining an evidence base for reform of design instruction is far from a trivial endeavor. While several researchers have collected and analyzed process data collected in the context of design courses (Agogino et al. to appear; Dong et al. 2004; Hill et al. 2002), controlled experimentation in a design course is challenging because of the small number of groups within each course and thus low statistical power for analyses at the group level, the difficultly of evaluating learning, the subjectivity even in the judgment about the quality of the product, and the sheer volume of process data collected over the course of a whole semester or more (e.g., from course discussion boards). These problems are compounded when the project is different each year and when each subgroup is working on a different project or different part of a large project, so the products of the collaboration are not directly comparable.

Learning in engineering design courses has some features in common with Problem Based Learning (PBL) in the medical domain (Hmelo-Silver, 2004) in terms of its intended aims. It also shares many of the methodological difficulties that make it challenging to build up an evidence base to guide the effective design of such courses. One lesson that has been a consistent theme through much research on PBL (Hmelo-Silver, 2004; Faidley et al., 2000) is that its quality for supporting learning depends largely on the quality of the support offered by an expert facilitator, who is constantly present to keep the group interactions moving in a productive direction. Unfortunately, such an expert facilitator is not available to students in engineering design courses during the majority of their interactions, since these typically occur outside of regular class time.

Part of achieving effectiveness in collaborative design projects is learning to structure the process. A major problem student design teams face is learning how to collaborate, how to share ideas, and how to divide responsibilities (Tonso 2006). As mentioned, most team meetings occur without the presence of an instructor. With no structure or process explicitly in place, a design meeting can quickly turn into a social gathering where little is accomplished.

To the extent that lessons learned in a PBL context carry over to engineering design courses in which much of the learning activity occurs without an instructor present, there is reason to doubt whether this mode of instruction is as effective as it could be. Moreover, there is reason to hypothesize that a productive direction for investigating possible improvements would be to structure design courses in such a way as to enable instructors to play a greater facilitation role in the learning.

In light of this hypothesis, our work has been motivated by a desire to address three important research questions: 1) To what extent can we find evidence that the absence of an instructor/facilitator during much of the learning activities that are part of an engineering design course is impeding learning? 2) To what extent can we find evidence that strategic support offered by instructors would improve learning in project courses? And 3) To what extent can we find evidence that current technology is capable of offering instructors of project courses needed insights into group processes so that they have the opportunity to offer this strategic support?

\section{RESEARCH CONTEXT: THE RPCS COURSE}

The testbed course for our investigations, Rapid Prototyping of Computer Systems (RPCS), draws students from Computer Science, Electrical and Computer Engineering, Industrial Design, Human-Computer Interaction, and Mechanical Engineering. The RPCS course teaches students 
about the design of real-world systems for industry sponsors. The class has created solutions for emerging needs such as pervasive computing infrastructures (IBM), a GM Companion Car-Driver Interface, context-aware cell phone, SenSay (Krause et al. 2006), and other novel projects (Siewiorek et al. 1998).

\section{Course Structure}

The class is divided into three phases: conceptualization, detailed design, and implementation. Each phase lasts roughly four weeks and culminates in an oral presentation and a teamproduced written report that are given to the external clients. Students must define the functionality required at the end of each phase and each team determines how to deliver the functionality as the phase progresses. The experimental study reported later in this paper took place during the third part of the course in the Spring 2006 semester.

At the beginning of the semester, the students visit the enduser workplace to conduct observations and surveys. Next, they generate a baseline scenario. After that, they create a visionary scenario describing how technology might improve current practice. After developing an architecture of the prototype from many perspectives (e.g., electronic, software, mechanical, and user interaction), students begin the detailed design phase doing further planning on the various subsystems of the prototype. Each subsystem design team is composed of representatives from each of the disciplines. Furthermore, members of each subteam serve as liaisons to other subteams to ensure integration of the functions into a final product. The teams meet regularly for short, technically-focused meetings intended to solve specific problems and identify the impediments that need to be resolved. The class also meets as a group to identify barriers to progress posed by other teams. These meetings provide frequent updates on project status. During the implementation phase, students integrate the subsystems into a final product. The architecture is reviewed with the end user; detailed design is performed; components are ordered, tested, and evaluated; application hardware and software are implemented; subsystems are integrated; and finally the entire system is tested. Oral presentations, demonstrations, and comprehensive written reports are produced monthly to elicit feedback from the end user. At the end of the four month course, a prototype is available for field evaluation.

\section{Course Infrastructure}

In the RPCS course, students coordinate their efforts throughout the semester in a groupware environment known as the Kiva (http:/thekiva.org) (Finger et al. 2006a). The Kiva is a web-based, asynchronous collaboration tool that was first prototyped by the students in the RPCS course in 2003 under the auspices of an NSF CRCD Grant. The core interaction of the Kiva combines aspects of both email and bulletin boards to keep threaded discussions intact. Students can post documents, diagrams, conversations, meeting notes, notes to self, task assignments, and so on. The discussion pages are designed to feel like a chat session in which students respond easily to one another. For the rapid prototyping course, we have incorporated a worklog for students to track time spent, reflect on work, and plan for the coming period. Time and task can be consolidated by team and individual. Periodically, we post reflective design questions in the weekly log. The group correspondence in the Kiva provides us with important insights into group functioning.

While the Kiva captures data that would be valuable to instructors for gaining insights into group functioning, the sheer volume of correspondence is far too great for an instructor to keep up with. Typical Kivas have many thousands of posts organized into hundreds of threads. For example, last Spring's RPCS course had 692 topic threads, each with an average of about 10 posts per topic. The students posted 1,244 files and they were still posting even after the class was officially over. From coding the conversations on the Kiva, it is apparent that most team discussions take place through the Kiva rather than in email; if a private email conversation results in something the whole team should know, the conversation is posted to the Kiva. A separate database is created for each class or research group that requests a Kiva, but within a Kiva, all members have access to essentially all the data. One indication of the success of the software is that 14 of the 20 class Kivas that have been created were requested by faculty because students in their class demanded to use it, rather than Blackboard, the official Carnegie Mellon course management software. Part of our technical goal is to leverage this resource using technology to make an assessment about student productivity from their conversational behavior in this on-line environment.

\section{EVIDENCE OF NEEDED SUPPORT}

In pursuit of an answer to our first research question above, we have conducted a qualitative analysis of the conversational data in the Kiva for the RPCS 2006 course. Our findings offer some support for our suspicion that valuable opportunities for student learning are being lost in the absence of an instructor to support the learning processes (Koschmann et al., 2005). Using patterns of typical information requests extracted from a separate corpus of collaborative learning interactions, we were able to automatically extract 108 information requests such as, "Um i dont know if it just me but i did see GPS sensor section in there and i posted stuff on GPS on wed am i missing something ?” Roughly half of these information requests were related to team coordination. However, the other half were more substantially related to design issues or basic skills required to carry out the work. We then examined the threads where these information requests were posted to see how team-mates responded. We found that a substantive response was forthcoming in only 73 of these cases, or roughly 68\%. In other cases, we found no evidence of a response to an information request, and occasionally students 
received a dismissive response. For example, here is a case where a student posted a substantive information request:

"I've looked at the spreadsheet that [a team mate] posted above and I have a question about my part. I know that I am in charge of the math models but I really don't know much about the dashboard computations (distance, energy used by appliances, cars generating pollution, energy produced by solar power). Is it possible if any of you can help me in the beginning so that I can complete this part?”

Rather than offering the help this student would have needed to be able to gain the skills to do this part of the work, the team reassigned the task to a different team member who already possessed this expertise. If the instructor had been aware of this incident, this would have been an excellent opportunity to step in and encourage students to take an approach that would maximize student learning rather than productivity.

\section{PILOT STUDY: EVIDENCE BASED DESIGN}

Even if the current structure of most engineering design courses does not maximize student learning, it is not clear a priori that the situation could be improved without a major course redesign. Thus, we address the second of our two research questions, which asks to what extent student learning in design courses can be enhanced with strategic instruction offered by instructors on an as-needed basis without requiring an instructor to be present for all group work. Thus, we set out to address this question with a small experimental study.

\section{Measuring Learning}

Part of the challenge of conducting this research is operationalizing the learning that takes place in design courses. In this section we introduce one of the important measures for assessing group functioning which we use in the experimental study. Note that we do not claim that this assessment captures all of what we hope students will learn in engineering design courses. Rather, it is simply an instrument we can use to measure a difference in instructional effectiveness between two alternative approaches.

The operationalization of learning we use of is motivated by an important problem in engineering design. Errors in design are a drain on group resources, and the later in the process they are detected, the more expensive they are to fix (NIST 2002). The majority of errors are introduced during the early design stages of requirements gathering and architectural design, and then detected during system integration (NIST 2002). Preventing and detecting errors before the system integration stage has a large payoff in terms of time and cost savings. We refer to these errors that occur as a result of a breakdown in communication as design escapes. We operationalize learning in this study in terms of student ability to identify and document design escapes as they occur during a small design task.

Design escapes have their origin in parallel design activities having conflicting assumptions, which is a typical occurrence in large, multi-disciplinary collaborations both in industry and in project courses such as RPCS. Often they are due to a failure to communicate a design decision made in one domain that affects another domain. For example, in the first wearable computer developed by the RPCS class, the electronic engineers built a reset button on the electronic board but had failed to notify the mechanical engineers and the industrial designers. As a result, the housing for the computer had to be modified at the last minute, the reset button had to be relocated on the electronics board, resulting in a metal-to-metal contact shorting out pins on the memory chip. We estimated that this design escape alone added $20 \%$ in the number of person hours and an additional $20 \%$ in cost to the project.

Teaching the ability to identify and document design escapes is important particularly because it has been difficult to circumvent the occurrence of design escapes in the RPCS course through more standard approaches to increasing instructor supervision or student involvement across teams. For example, as a consequence of the experience mentioned above, we instituted liaisons between design groups. Each design group is responsible for sending a liaison to every other design groups' meeting with the sole purpose to listen for design decisions that affect their group and to inform the other group if they are making decisions incompatible with decisions that had already been made by their group. However, students often put a low priority on attending another group's meeting. In addition, at the weekly leaders' meetings students give summaries of what has happened in the past week; however, since students are inexperienced they often fail to recognize that they have made a conflicting decision. As a consequence, one labor-intensive requirement is that faculty must attend some of the design group meetings to provide in-process feedback to the students so that they not only learn but have the opportunity to make corrections in real time. Without this inprocess feedback, the time to discover problems is greatly expanded, threatening both the scope and successful, timely completion of the project.

Facilitating the process of identifying and documenting design escapes is an important part of what we hope to achieve with the in process feedback technology we are working on developing. We propose to accomplish this by making the collaboration process more transparent to participants both at the project management level and at the project execution level. As we have taught the RPCS course from year to year, we have experimented with the Orthogonal Defect Classification (ODC) methodology, which was developed by IBM Watson Research Center over a number of years (Chillarege 1992). It has been deployed at over ten IBM sites with over 4000 developers and testers with an annual savings estimated at over \$100M. ODC captures the semantics of each defect and links their statistical distribution to points in the design process. Analysis of defects 
contains rich information on both the design process and the object being designed. Different defect classes arise at different points in the design process. Collecting data on the point of origin of a defect and its point of discovery provides a statistical profile of the design and defect discovery process. For each defect discovered, the designer classifies it on multiple dimensions including: source (where the defect is located), target (what was fixed), trigger (how the defect was detected), etc. As design teams enter information into ODC, they receive immediate feedback with respect to the statistical distribution of defects being discovered during the current design with respect to previous historical designs. If discovery of defects of certain classes is under represented with respect to the point in the design process, ODC makes suggestions to the design team about reallocating resources. We have reviewed several generations of the RPCS course and interviewed student designers to extend the ODC classifications to include electronic, mechanical, and human interaction.

Thus, monitoring the occurrence of design escapes, noting both when they first occurred and when they were detected, how, and by whom, is a way we propose to evaluate the effectiveness of an instructional approach in a project based course such as RPCS. While the time that a design escape was caused is not immediately obvious upon detection of the fault, it is frequently possible to track down the source of design escapes, at least to within a week of their genesis, which is a fine enough grain size for our analysis. We have created a modified version of the ODC, which we call the Design Escape Detector (DED), to help students identify and repair design escapes. The DED is designed to encourage cross team communication, so that as the students identify and classify defects as part of their weekly reports, they begin to internalize the causes of design escapes so they can design to prevent them in future phases. The list of design escape attributes encoded in DEDs, which we refer to below as ODC forms, includes:

- phase found: Conceptualization, Detailed Design or Implementation;

- class: Error of Omission or Error of Commission;

- trigger: Review and Inspection, Unit Test, System Verification or Field Test; Review and Inspection includes formal software reviews and evaluation. Unit test covers directed tests of isolated features. System Verification and Field Test involves integrated subsystems operating together

- source: Software, Hardware, or Physical. Source has subattributes which indicate whether the escape came from a component provided by a Vendor, resulted from Reuse from an earlier version, or is a New design escape

- impact: Critical, Major, Minor; The impact attribute has subattributes indicating whether the design escape has an impact on Performance, Reliability, Lost Objective, Usability, or Maintainability

\section{Method}

In Spring of 2006, we ran a pilot study in RPCS to begin to evaluate the potential learning benefit in terms of teaching how to identify and document design escapes that might result from supporting communication processes in engineering design courses. The preliminary results show promise that 1 ) we can develop assessments that are sensitive enough to show measurable learning in design courses, 2) our experimental paradigm provides a framework in which we can demonstrate a measurable effect of an experimental manipulation in comparison to a control condition, and 3) supporting communication is likely to lead to a learning benefit for students that can be detected to some extent even several weeks after the targeted instruction was offered.

Tasks. For our study, we injected two mini-design projects into the course as pre and post-test activities for student teams. Both of these tasks are a microcosm of the course in that student teams go through the entire design and development process of a project within a single class period, thus allowing the instructors and TAs to observe all teams as they are working and make observations about their process. Prior to the pretest activity, students were given a lecture on Orthogonal Defect Classification. They were introduced to the forms they would use to report faults. The pretest exercise was their first opportunity to apply this knowledge during a development activity. The experimental manipulation took place in the week subsequent to the pretest activity when student teams were individually offered feedback on their process and usage of orthogonal defect classification during their pretest activity. They then had four weeks to use orthogonal defect classification in their regular project work. In the fifth week, students participated in a second design microcosm activity so that we could observe. Again instructors and TAs observed student process and usage of Orthogonal Defect Classification during the post-test activity. For both tasks, students were given a certain amount of play money to purchase materials that were available from a store. Student teams were given their design challenge and then had to plan together how to most strategically spend the money they had to meet their goals. They then had to go through the whole process of ideation, design, implementation, and testing.

- $\quad$ Task 1 - The Airplane task: For the airplane task, students were give the task of designing a simple glider airplane that would be evaluated by launching it from a stage in an auditorium and measuring how long it stayed in the air.

- Task 2 - The Egg Drop Task: For the egg drop task, students were given the challenge of building a protective case for an egg such that when it was dropped from the side of a stairwell down two flights, it would not break, or even crack.

Hypothesis. In the pilot study we tested the hypothesis that by increasing the amount of targeted feedback offered to students about the details of their in-progress process and product, we 
could increase their learning and possibly improve their final product. If this is true, it supports the potential benefit that an on-line assessment system would offer to a design course since it would enable instructors to keep a closer eye on group processes throughout the semester, and to step in when needed to offer targeted feedback to students.

Outcome variables. We evaluated students' pre-test and posttest performance using The Airplane problem and The Egg Drop Problem. With reference to the ODC forms submitted by student teams and instructors, we evaluated team performance in terms of 1) thoroughness of identifying faults 2) success in avoiding faults 3) correctness of filling out ODC forms, 4) quality of explanation of why their design is good 5) performance measures related to their design (e.g., egg protection, on target, etc.)

Experimental Manipulation. We tested our hypothesis by means of a simple two condition manipulation in which we offered targeted feedback after the pre-test exercise to the experimental group and generic feedback to the control group. The class was divided into 8 pre/post test groups. Groups were randomly assigned to conditions. We counter-balanced the pre and post test exercise to control for any differences in task difficulty and opportunities for learning between the two exercises. The feedback was designed to address the first three learning objectives. All students were offered generic feedback related to these 3 objectives, focusing on issues that were relevant broadly across groups. The feedback for both groups included a fully worked out example of a correctly filled out ODC form, which is known to be an effective instructional tool, especially at early stages of skill acquisition (Atkinson, 2003; Renkl \& Atkinson 2003; Renkl, 2002). For the targeted feedback, we inserted specific pointers to observations we made about their group's process from the pre-test exercise. And the correctly filled out ODC form was a corrected version of one of the forms their group submitted, whereas in the case of the control condition, it was an example that best exemplified a correction of a typical error encountered in multiple groups.

Mediating variables. Using the Kiva data, we are able to analyze on-line conversations throughout the month-long period between the pre-test exercise and the post-test exercise. However, we have not yet analyzed this data from the Spring 2006 pilot study in depth beyond the automatic analyses we present later in the paper.

Results. Our preliminary results suggest that our experimental manipulation had an effect on student learning. In the pre-test exercise, we found that almost without exception, students were not able to use the ODC forms successfully. There was almost no overlap between the issues students identified as faults and those the instructors identified as faults. Furthermore, students did not correctly fill out the ODC forms. There was no difference between conditions on pretest performance. On the post-test, we saw the biggest improvement in the experimental group. Using a binary logistic regression, we determined that the proportion of correctly filled out ODC forms to incorrectly filled out forms was marginally higher in the experimental group $(\mathrm{p}<.1)$. Furthermore, we observed a greater overlap in issues raised on ODC forms between student groups and instructors within the experimental group, especially in connection with the Airplane exercise.

While these results are only suggestive due to the lack of statistical significance, we expect that this lack of significance is primarily due to a type II error since one group in each condition did not show up for the post-test, leaving us only 3 groups in each condition. We plan to address this issue in future studies by collecting data over multiple semesters and by making repeated assessments throughout the semester, such as with the reflective exercises embedded in work logs, as discussed above. Our data suggests that the development of an infrastructure that offers project class instructors a portal into the day-to-day inner workings of the sub-groups within these classes is worth pursuing.

\section{TOWARDS ON-LINE ASSESSMENT OF ENGINEERING DESIGN LEARNING}

With some evidence that students would benefit from strategic support from instructors, we must then address the third of our research questions, which is the extent to which we can use available technology to offer instructors the insights needed to offer this strategic support. Our goal is to develop an infrastructure that is able to address the assessment dilemma in project-based courses. In other words, the infrastructure we are working towards developing is meant to facilitate in-depth and timely assessment during regular learning activities rather than taking time away from classroom activities - similar in spirit to our related work on assessment during instruction in middle school math (Rosé et al. 2005b; Razzaq et al. 2005). Webb and colleagues (Webb \& Mastergoerge 2003; Webb et al. 2002) have demonstrated strong correlations between patterns of collaborative discourse and learning, and we draw from this foundational work.

Our technical approach is to use machine learning to build models that can identify meaningful patterns in the conversational behavior of students. It can then use these to form an assessment of the student's course behavior and thus provide a useful report to the instructor. Machine learning algorithms can learn mappings between a set of input features and a set of output categories or numeric predictions (such as a regression model). They do this by examining a set of hand coded "training examples" that exemplify each of the target categories. The goal of the algorithm is to learn rules by generalizing from these examples in such a way that the rules can be applied effectively to new examples. Once the input features have been set, a large number of training examples is then typically hand-coded. Toolkits such as Weka (Witten \& Frank, 2005) provide a large number of alternative machine learning approaches. 
As a proof of concept, we are developing a browsable reporting system for project course instructors that makes an assessment about student productivity based on a model trained on features extracted from student conversation behavior on a groupware system. We chose productivity as an outcome measure because it was formally assessed by instructors in the RPCS 2006 course, and thus we have access to that data in addition to the conversational data on the Kiva. However, ultimately we plan to use a measure of student learning as the outcome measure. The work we report here is meant to be a proof of concept for the methodology.

Productivity grades are assigned 3 times in the semester and are an important means of communication between instructor and students about standing within the course. We have investigated how evidence of student productivity extracted from student on-line behavior correlates with instructor judgment of productivity based on the instructor's human understanding of the worklogs, observations in class, assessment of group product, and insights gained from weekly meetings. Our finding is that shallow indicators that have been used in prior state-of-the-art work such as number of posts, number of attachments, and length of posts (see, for example Kay et al., 2006a; Kay et al., 2006b; Kreijns and Kirschner, 2004) have no significant correlation with an instructor assigned productivity grade in our data. However, using a model constructed with the TagHelper toolset developed in our previous research (Donmez 2005), we have been able to build a model that can make a prediction about student productivity based on data that was not used in training the model $(\mathrm{R}=.63)$. While we are continuing to work to improve this prediction accuracy, we believe these preliminary results show promise than an automatic analysis of on-line communication behavior of groups can provide instructors with valuable early warning signs that some groups or certain students within groups require some additional instructor support.

\section{Evaluation Details}

We ran our evaluation using 1157 posts from the RPCS spring 2006 course. We divided the data into collections of posts posted by an individual student on a specific week of the course. Altogether we assembled 476 such posts. Associated with each of these collections was a target productivity grade, which was assigned by the instructor. Productivity grades were assigned by the instructor 3 times in the semester. Thus, the grade associated in this data with an individual week was the grade assigned to the student for the segment of the course that week was part of. Since student productivity may vary from week to week throughout the semester, we consider this target assessment to be somewhat noisy. However, it is the best objective measure we have for individual student productivity in the course.

From this raw data, we extracted a number of linguistic features using TagHelper tools, which can be downloaded from http://www.cs.cmu.edu/ cprose/TagHelper.html. These features included stemmed unigrams (i.e., single word stems), and bigrams (i.e., pairs of word stems occurring adjacent in the text). We did not include words that occurred less than 5 times in the corpus or typical function words such as prepositions or determiners. In addition to this we included features extracted by using the Pennebaker's Linguistic Inquiry and Word Count (LIWC) (Pennebaker et al., 2001). Using a support vector machine regression model included in the Weka machine learning package (Witten \& Frank, 2005), we achieved a correlation coefficient of .63 using a 10 fold cross-validation methodology so that the correlation was evaluated over separate data from what it was trained on. Adding additional features such a number of posts, number of active days during the week, or number of files committed did not improve this correlation.

Note that while in some sense the features used in our trained model could still be considered "shallow indicators," they provide more detailed information about student interactions than the indicators from prior work that do not consider the content nor the structure of student conversational behavior. Interestingly, some of the most predictive linguistic features were social in nature. For example, we observed that words such as "thanks," "hi" and "please" ranked among the top attributes in the feature space. This might suggest that more polite and friendly groups makes the group a more attractive environment to work in, thus students exhibit less social loafing behavior as implied by the Collective Effort Model by Karau and Williams (1993).

Our long term goal is to develop text processing technology that will be capable of preparing reports for instructors that will offer them an abbreviated view, or summary, of the vast amount of conversational behavior on the Kiva, with the capability of allowing them to drill down to get more detail about the episodes that appear to be of interest. This form of conversation summarization, which is a relatively new area of computational linguistics, builds on a long history of expository text summarization. While typical applications of summarization technology extract the most contentful sentences from a document or from multiple documents reporting about the same event (Kupiec, Pedersen, \& Chen, 1995; Gong \& Liu, 2001), the goal of conversation summarization is to capture what people consider important in a conversation. Behavioral studies about dialogue summarization show that what people consider important to include in a summary about a dialogue may include aspects of the nature of the conversation in addition to a condensed version of the information that was communicated. Technology development for conversation summarization has frequently focused either on topic segmentation or on dialogue act identification (Zechner, 2001). For example, topic segmentation has been used for separating multiple threads in an IRC conversation and then doing extractive summarization within threads (Zhou \& Hovy, 2006) or meeting summarization in which topic segmentation divides regions of the conversation in which separate agenda items are being discussed. Dialogue act tagging has been used to identify places in the conversation 
where certain types of conversational events occurred (Zechner, 2001). We have begun to work on both of these problems (Arguello \& Rosé, 2006a; Arguello \& Rosé, 2006b; Arguello \& Rosé, 2006c; Rosé et al., 2005; Donmez et al., 2005).

One can think of these browsable summaries as an alternative to current conversation visualization work (Donath, 2002; Popolov, 2000; Sack, 2000), however they are quite different in terms of approach. While the goal is similarly to provide a way of quickly getting a sense of what is happening in an on-going conversation, conversation visualizations tend to display a conversation in much greater detail than conversation summaries. We believe these approaches could be combined and this is an ongoing topic in our research.

Figure 1 displays a screen shot from our prototype system. Note that the system predicts a high score for people who received a poor productivity grade from their instructor and a low score if they received a good grade. We refer to this indicator as "the Social Loafing Tendency," or the extent to which students behave like "free riders" in their group.

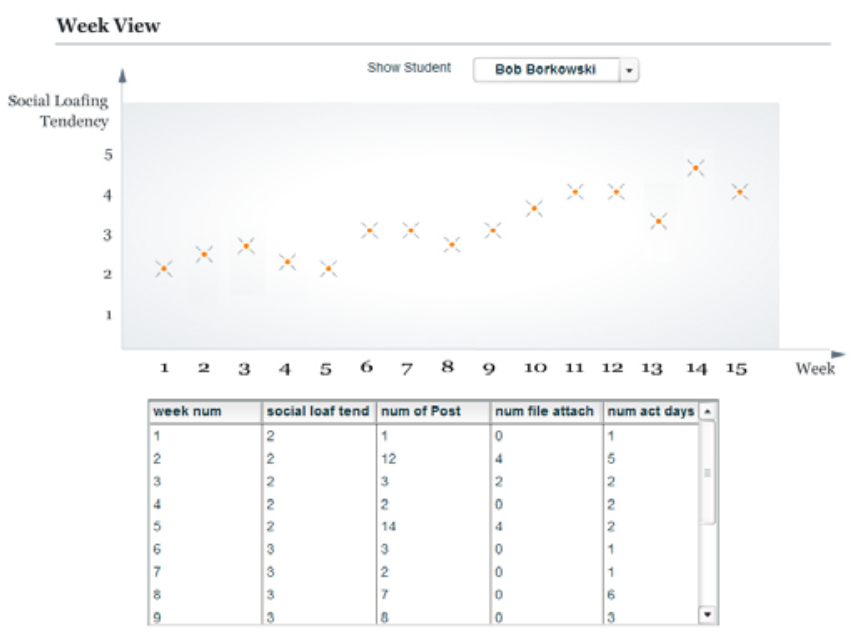

Figure 1. Prototype Interface with Predictions Displayed in the Upper Panel

Our prototype reporting system is meant to alert instructors to groups that may require more instructor intervention to keep the group on track by providing an overview of each student's productivity performance over time. In our current work, we are working towards making this interface "browsable" by allowing instructors to click on the predictions for a specific week and "drill down" to the raw communication data that was used to form the prediction.

\section{RELATED WORK AND CURRENT DIRECTIONS}

This is not the first effort to extract assessment related information from on-line discussion behavior, and we are still far from our ultimate goal. Here we discuss some prior related work and some of our current directions. Agogino, Song, \&
Hey (2006) show with their work that text processing approaches such as Latent Semantic Analysis (LSA) (Foltz et al., 1998) can be used to calibrate patterns found in corpora of collaborative interactions with group outcomes. One main claim from their analysis is that it is important for groups to generate a lot of ideas and then converge at each stage before moving on (Agogino et al. 2006; Dong et al. 2004; Hill et al. 2002). This finding is consistent with other work on argumentative knowledge construction in which highlights the importance of group members directly addressing their disagreements rather than talking at cross purposes or engaging in quick consensus building (Weinberger 2003). One drawback of the approach taken by Agogino and colleagues is that their analysis method does not give them the ability to discover how the teams came to a consensus. Their work does not study how the features of the collaborative process affect either learning or the products produced during the collaboration. They present some evidence supporting the link between the process information extracted using their methods and the product outcomes they evaluate. A more detailed analysis of the collaborative process data might reveal stronger links between process and product, which is what we seek to identify in our research.

Issues related to collaborative process data that are closer to our current and long term concerns include the following questions: 1) to what extent are participants reflecting critically on their design process and the effects of their process on other subgroups, 2) about what issues and in what manner are subgroups communicating with other subgroups, 3) to what extent are subgroups communicating effectively with their supervisors/instructors, especially about intra-group and intergroup struggles? While recent work reviewed by Kreijns and Kirschner (2004) relies primarily on shallow analyses of collaborative learning interactions, such as the total number of words contributed by each participant, we expect that deeper analyses of the interactions will be required in order to accomplish our goals. Thus, we propose to build upon our own recent work in which language technologies are employed and extended in order to allow more of the freeform interactions between participants to be analyzed in a meaningful way (Donmez et al. 2005; Rosé et al. 2005).

\section{CONCLUSIONS}

In this paper we have explored the use of text processing technology for on-line assessment in an engineering design project class. We have offered some preliminary validations of our approach in the form of a small classroom study conducted in an engineering design project course as well as a corpus based experiment where we have demonstrated our ability to make predictions about student productivity from their behavior in an on-line groupware system.

We acknowledge that our current approach is limited in that it only takes into consideration what happens in the on-line setting, while most of the interesting design work is accomplished during in-person meetings. However, in our 
current work we are exploring the possibility of recording inperson meetings so that we could use this data to make predictions, about student productivity, the quality of their design process, and about their learning.

\section{ACKNOWLEDGMENTS}

This research was supported in part by National Science Foundation grants EEC-0203448 and SBE0354420.

\section{REFERENCES}

Adams, R.S., Turns, J., Atman, C.J., 2003, “Educating Effective Engineering Designers: The Role of Reflective Practice,” Design Studies, 24(3), 275-294.

Agogino, A., Song, S., Hey, J., "Triangulation of Indicators of Successful Student Design Teams,” International Journal of Engineering Education, 22(3), 617-625.

Arguello, J. \& Rosé, C.P., 2006a, "Museli: A Multi-source Evidence Integration Approach to Topic Segmentation of Spontaneous Dialogue,” Proceedings of the North American Chapter of the Association for Computational Linguistics (short paper).

Arguello, J. \& Rosé, C.P., 2006b, “Topic Segmentation of Dialogue,” Proceedings of the NAACL Workshop on Analyzing Conversations in Text and Speech.

Arguello, J. \& Rosé, C.P., 2006c, “InfoMagnets: Making Sense of Corpus Data," Companion Proceedings for the North American Chapter of the Association for Computational Linguistics (NAACL ’06).

Atman, C.J., Chimka, J.R., Bursic, K.M., \& Nachtmann, H.L., 1999, "A Comparison of Freshman and Senior Engineering Design Processes,” Design Studies, 20(2), 131-152.

Atkinson, R., 2003, “Transitioning From Studying Examples to Solving Problems: Effects of Self-Explanation Prompts and Fading Worked-Out Steps,” Journal of Educational Psychology, 95(4).

Chillarege, R., Bhandari, I. S., Chaar, J.K., Halliday, M.J., Moebus, D.S., Ray, B.K., Wong, M., 1992, “Orthogonal Defect Classification-A Concept for In-Process Measurements," IEEE Transactions on Software Engineering, 18, 943-956.

Dong, A., Hill, A., Agogino, A.M., 2004, “A document analysis technique for characterizing design team performance. Journal of Mechanical Design, 126(3), 378385.

Donath, J., 2002, “A semantic approach to visualizing on-line conversations," Communications of the ACM, 45(4), 4549.

Donmez, P., Rose, C. P., Stegmann, K., Weinberger, A., Fischer, F., 2005, "Supporting CSCL with Automatic Corpus Analysis Technology,” Proceedings of Computer Supported Collaborative Learning.
Dutson, A.J., Todd, R.H., Magleby, S.P. \& Sorenson, C.D., 1997, “A Review of Literature on Teaching Engineering Design through Project-oriented Capstone Courses,” Journal of Engineering Education, 86(1), 17-28.

Faidley, J., Evensen, D. H., Salisbury-Glennon, J., Glenn, P. \& Hmelo, C., 2000, "How are we doing?: Methods of Assessing Group Processing in a Problem-Based Learning Context,” In Evensen, D. H., and Hmelo, C. E. (eds.), Problem-Based Learning: A Research Perspective on Learning Interactions, Erlbaum, Mahwah, NJ, 109-135.

Finger, S., Gelman, D., Fay, A., Szczerban, M, Smailagic, S. \& Siewiorek, D.P., 2006, "Supporting Collaborative Learning in Engineering Design,” International Journal of Expert Systems and Applications.

Foltz, P. Kintsch, W. \& Landauer, T., 1998, “The measurement of textual cohesion with latent semantic analysis,"

Discourse Processes, 25, 285-307

Gong, Y. \& Liu, X., 2001, “Generic Text Summarization Using Relevance Measure and Latent Semantic Analysis," Proceedings of ACM SIG-IR 2001.

Hill, A., Dong, A. \& Agogino, A.M., 2002, “Towards computational tools for supporting the reflective team," $A I$ in Design 2002, Gero, J. (ed.) Kluwer, 305-325.

Hmelo-Silver, C. E., 2004, "Problem-Based Learning: What and How Do Students Learn?” Educational Psychology Review, 16(3), 235-266.

IBM Corporation, "Rational Rose Technical Developer,” http://www-306.ibm.com/software/awdtools/ developer/technical/

Karau, S. \& Williams, K., 1993, “Social Loafing: A MetaAnalytic Review and Theoretical Integration,” Journal of Personality and Social Psychology, 65, 681-706.

Kay, J., Maisonneuve, N., Yacef, K. \& Reimann, P., 2006a, The Big Five and Visualization of Team Work Activity, University of Sydney Technical Report 581, January 2006.

Kay, J., Maisonneuve, N., Yacef, K. \& Reimann, P., 2006b, Wattle Tree: What'll It Tell Us?, University of Sydney Technical Report 582, January 2006.

Koschmann, T., Zemel, A., Conlee-Stevens, M., Young, N., Robbs, J. \& Barnhart, A., 2005, “How Do People Learn? Members' Methods and Communicative Mediation," In Bromme, Hesse, and Spada (eds.) Barriers and Biases in Computer Mediated Knowledge Communication and How they Can be Overcome, Dordecht, Netherlands: Kluwer.

Krause, A., Smailagic, A. \& Siewiorek, D.P., 2006, “ContextAware Mobile Computing: Learning Context-Dependent Personal Preferences from a Wearable Sensor Array,” IEEE Transactions on Mobile Computing, 5(2), 113-127.

Kreijns, K. \& Kirschner, P.A., 2004, “Designing Sociable CSCL Environments,” In Strijbos, Kirschner, \& Martens 
(eds.) What We Know About CSCL: And Implementing It, Dortrecht: Kluwer Academic Publishers.

Kupiec, J., Pederson, J., \& Chen, F., 1995, “A Trainable Document Summarizer,” Proceedings of ACM SIG-IR.

Krause, A., Smailagic, A., Siewiorek, D., 2005, “ContextAware Mobile Computing: Learning Context-Dependent Personal Preferences from a Wearable Sensor Array,” IEEE Transactions on Mobile Computing.

NIST, 2002,The Economic Impact of Inadequate Infrastructure for Software Testing, RTI, Research Triangle Park for the National Institute of Standards \& Technology, Report 0203.

Pennebaker, J.W., Francis, M.E., \& Booth, R.J., 2001, Linguistic inquiry and word count (liwc2001). Mahwah, NJ: Erlbaum Publishers.

Popolov, D., Callaghan, M., \& Luker, P., 2000, “Conversation space: Visualizing multi-threaded conversation,” Paper presented at the AVI, Palermo, Italy.

Razzaq, L., Feng, M., Nuzzo-Jones, G., Heffernan, N.T., Koedinger, K. R., Junker, B., Ritter, S., Knight, A., Aniszczyk, C., Choksey, S., Livak, T., Mercado, E., Turner, T.E., Upalekar, R, Walonoski, J.A., Macasek, M.A. \& Rasmussen, K.P., 2005, “The Assistment Project: Blending Assessment and Assisting,” In C.K. Looi, G. McCalla, B. Bredeweg, \& J. Breuker (Eds.) Proceedings of the 12th Artificial Intelligence In Education, 555-562. Amsterdam: ISO Press.

Renkl, A., 2002, “Learning from worked-out examples: Instructional explanations supplement self-explanations," Learning \& Instruction, 12, 529-556.

Renkl, A., and Atkinson, R., 2003, "Structuring the transition from example study to problem solving in cognitive skill acquisition: A cognitive load perspective,” Educational Psychologist, 38(1), pp15-22.

Rosé, C., Donmez, P., Gweon, G., Knight, A., Junker, B., Cohen, W., Koedinger, K. \& Heffernan, N., 2005,

"Automatic and Semi-Automatic Skill Coding with a View Towards Supporting On-Line Assessment,” Proceedings of $A I$ in Education '05.

Sack, W., 2000, “Conversation map: An interface for very large-scale conversations,” Journal of Management Information Systems, 17(3), 73-92.

Siewiorek, D.P., Smailagic, A., Bass, L., Siegel, J., Martin, R. \& Bennington, B., 1998, “Adtranz: A Mobile Computing System for Maintenance and Collaboration,” Proceedings 2nd IEEE International Conference on Wearable Computers, Pittsburgh, PA.

Strijbos, J.W., 2004, The Effect of Roles on ComputerSupported Collaborative Learning, Maastricht: Datawyse boek - en graphische producties.
Tonso K.L., 2006, “Teams that Work: Campus Culture, Engineer Identity, and Social Interactions,” Journal of Engineering Education, 95(1), 25-37.

Webb, N., Nemer, K. \& Zuniga, S., 2002, “Short Circuits or Superconductors? Effects of Group Composition on HighAchieving Students' Science Assessment Performance,” American Educational Research Journal, 39(4), 943-989.

Webb, N. \& Mastergeorge, A., 2003, “The Development of Students' Helping Behavior and Learning in Peer-Directed Small Groups," Cognition and Instruction, 21(4), 362-428.

Weinberger, A., 2003, Scripts for Computer-Supported Collaborative Learning Effects of social and epistemic cooperation scripts on collaborative knowledge construction, PhD Thesis, University of Munich, Munich, Germany, http://edoc.ub.uni-muenchen.de/archive/ 00001120/01/Weinberger_Armin.pdf

Witten, I. H. \& Frank, E., 2005, Data Mining: Practical Machine Learning Tools and Techniques, second edition, Elsevier: San Francisco, ISBN 0-12-088407-0

Zechner, K., 2001, “Automatic Generation of Concise Summaries of Spoken Dialogues in Unrestricted Domains,” Proceedings of ACM SIG-IR 2001.

Zhou, L., Lin, C. \& Hovy, E., 2005, “A BE-based Multidocument Summarizer with Sentence Compression,” In Proceedings of ACL 2005 (MSE workshop). 